\title{
Analysis of back propagation and radial basis function neural networks for handover decisions in wireless communication
}

\author{
Payal Mahajan, Zaheeruddin
}

Department of Electrical Engineering, Jamia Millia Islamia University, India

\begin{tabular}{l}
\hline \hline Article Info \\
\hline Article history: \\
Received Apr 8, 2019 \\
Revised Mar 13, 2020 \\
Accepted Mar 31, 2020 \\
\hline Keywords: \\
Back propagation networks \\
Handover \\
Neural networks \\
Quality of service \\
Radial basis functions \\
\hline
\end{tabular}

\begin{abstract}
In mobile systems, handoff is a vital process, referring to a process of allocating an ongoing call from one BS to another BS. The handover technique is very important to maintain the Quality of service. Handover algorithms, based on neural networks, fuzzy logic etc. can be used for the same purpose to keep Quality of service as high as possible. In this paper, it is proposed that back propagation networks and radial basis functions may be used for taking handover decision in wireless communication networks. The performance of these classifiers is evaluated on the basis of neurons in hidden layer, training time and classification accuracy. The proposed approach shows that radial basis function neural network give better results for making handover decisions in wireless heterogeneous networks with classification accuracy of $90 \%$.
\end{abstract}

Copyright $\left({ }^{\circ} 2020\right.$ Institute of Advanced Engineering and Science. All rights reserved.

\section{Corresponding Author:}

Payal Mahajan,

Department of Electrical Engineering,

Jamia Millia Islamia University,

New Delhi, India.

Email: payal27mahajan@gmail.com

\section{INTRODUCTION}

Introduction to wireless communication. Wireless communication now a days is the part and parcel of our lives since it offers elasticity and freedom of moving from place to a different while not losing connectivity to the network. New interactive program services request rate of up to hundreds of megabits per second and so bigger bands of frequencies are needed to keep up these new elevated data rate services. On the contrary, to make mobile nodes move problem free, seamless handover is very important in these networks [1]. VHO (vertical handover) algorithms are necessary for the development of the next Generation HetNets i.e. heterogeneous networks [2]. Web consumers are assumed to possess worldwide affiliation within the future web supported Next Generation networks. The ascension in the user request for knowledge access actuated the employees too looked for improved Radio Access Technologies for delivering services to the consumers additional expeditiously at higher speed. The well-established Third Generation network like Universal Mobile Telecommunications System created it attainable to supply elevated quality level with giant coverage however might supported solely low to medium rate that is insufficient to accomplish applications that desires continuous internet connection [3].

For wireless communication, because of restricted information measure and getting a good service, it's vital to lift the channel reuse ratio, thus an accessible and effective handover formula ought to be developed [4]. Next gen wireless communication networks ought to guarantee the perpetually best connected $(\mathrm{ABC})$ to mobile user. The performance of wireless network is improved by using well-ordered handover technologies once user is moving from one cell to a different [5]. This new era of communication involves the interworking of the many technologies to confirm that the user is often connected with the world. Relying upon the sort of application the user is running, different handover decisions are taken as an example if a user is sharing knowledge on a social networking website, he would like to urge connected with 
the technology that give him with the identical facility with less latency and permit him to use the services ceaselessly equally once he desires to hold an ongoing call he would like to urge connected to those service [6]. Thus to permit the user to enjoy the continual services an improved handoff mechanism is needed. Figure drawn below shows the interworking of various technologies. A new design concept, i.e. the Heterogeneous Networks, was introduced in LTE to meet demand for both cellular network capacity and coverage. HetNets ' idea is the deployment of several smaller cells under macro cell area to extend coverage or increase capacity in some high - demand areas.

HetNets is a major change in the design of cellular networks, offering increase in network capacity and enhanced coverage. HetNets refers to multi - access networks with the same UE (i.e. LTE with Wi-Fi) and may refer to hierarchical sector structures where multiple cell structures with the same radio access standards are used (macro cell). Recently, due to the rapid development of the current Internet and mobile communications industry, mobile networking systems have been designed with greater infrastructure complexity and greater diversity of associated devices and resources, as well as more dynamic networking formations. In such emerging heterogeneous mobile networks (HetNets), there are a large number of technical challenges over the complicated system resources focusing on the efficient management, organization. The working of the efficient wireless communication system is the main focus these days. Many authors have contributed in the improvement and innovation of the wireless communication systems. In this work, we have suggested the use of Radial Basis and Back propagation neural tools for making handover decisions. The results are deduced from the Matlab functions and it is seen that RBF works efficiently than BPNN for making handover decisions in wireless heterogeneous networks. These three features are covered by the Heterogenous wireless Networks:

- Intelligent and seamless network - wide access - HetNet can improve the network switching procedures to deliver a seamless movement experience as users move between different Heterogeneous wireless networks. Furthermore, HetNets can provide devices with the necessary network information to enable authentication and access to various networks.

- Consistent Network Experience Quality - HetNets allow different networks to exchange information on loads such as data traffic and latency. Such capabilities enable a mobile device to identify the network that best suits the user's mobility needs. This results in a consistent quality of user experience across multiple networks and better distribution of loads across different networks.

- Innovative and Dynamic Resource Management - two types of intelligence are involved in dynamic resource management: spectrum agility and service agility. Reconfigurable radio with cognitive functions and opportunistic access to the spectrum needs spectrum agility. On the other hand, service agility requires virtualization techniques to circumvent computational networking issues. Collectively, dynamic resource management allows scalable, re - configurable and adaptable networks.

A structure of diverse wireless access networks is Heterogeneous Wireless Network is shown in Figure 1. With regard to coverage of network, technology, bandwidth, capacity, and network operator, VHO is done [7]. A Vertical Handoff (VHO) occurred in such an atmosphere where a mobile terminal (MT) with a number of network interfaces leave a wireless network and connects to other wireless network [8]. But on what basis to perform, where to perform, when to perform the Vertical Handover are the key questions that must be determined to carry out an efficient VHO for maximizing the supposed QoS (Quality of Service) and QoE (quality of experience) perceived by the user [1]. Selection of suitable handover decision attribute and assessment method play a vital role in this regard.

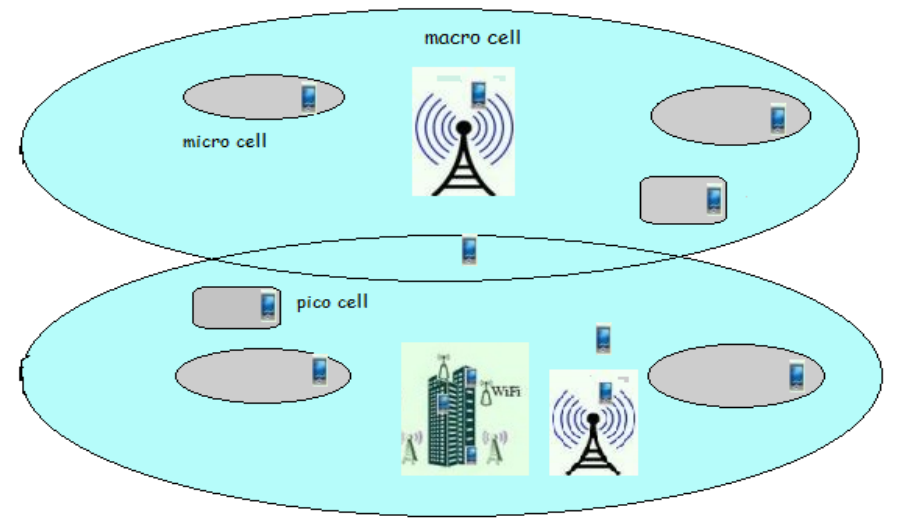

Figure 1. Heterogeneous wireless networks 
Handover refers to the procedure when an Mobile Terminal(MT) change its attachment point from one access point(AP) to other, as shown in Figure 2.

- Horizontal Handover

- Vertical Handover

Horizontal handover occur when a MT (mobile terminal) moves within the same wireless network from one cell to another. Horizontal handoffs [9] can also be known as Inter-BSC handoff, Intra-BSC handoff and Inter-Msc handoff in the cellular wireless system. The reasons for which horizontal handovers are done are: poor signal quality (RSS) or signal loss, assignment of bandwidth, load balancing and Mobile Terminal speed. The HHO (horizontal handover) process consists of three foremost steps: initiation of the handoff, assignment of the channel, and execution of the handover / connection transfer [4].

Vertical handover can be done in three phases called Network discovery, Handover decision, and execution of Handover. Network discovery is a process of finding the appropriate network in such a way that the network meets user preferences with the required service quality [2]. Too early handover results in redundant handover, while service quality is reduced as a result of late handover. Lastly, the transfer should be executed at an optimal time. Several algos have been anticipated for vertical handover (VHO) mechanisms on the basis of number of parameters, namely the received signal strength (RSS), connection costs, available bandwidth, and speed of the mobile station, service quality, and signal to interference noise ratio, guarantee delay of handover and battery consumption. The objective of the next generation communication systems is to offer users, a large range of options [10].

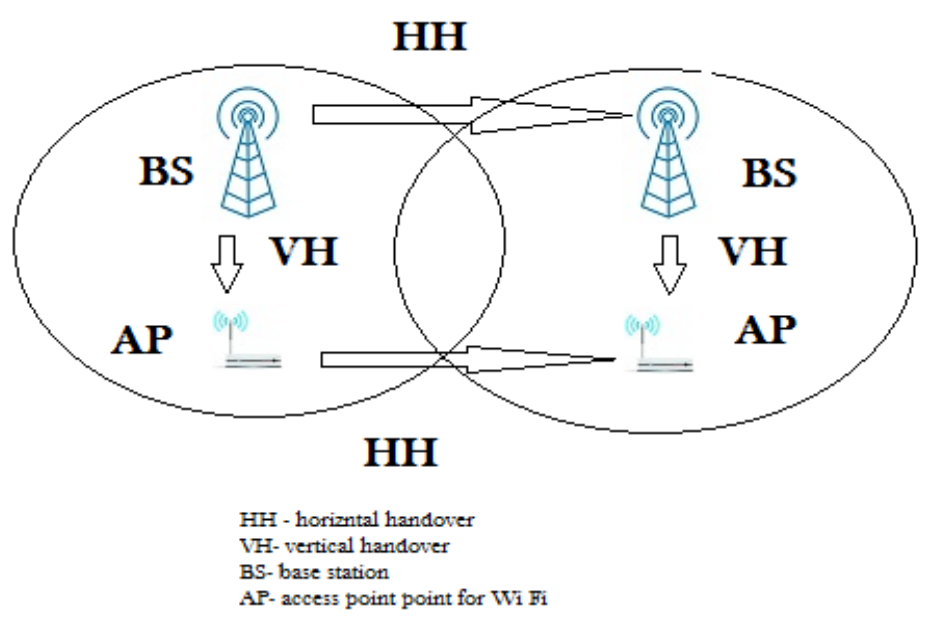

Figure 2. Handover in heterogeneous networks

\section{RELATED WORK}

Many authors have contributed in the field of wireless communication. Some of the work which helped us in this work are shown here. In [11], the authors gave a method by which selection of the optimum target network from the available networks can be done. This approach is designed to maximize the degree of customer satisfaction by selecting the "best" network among numerous candidate networks as the handover target network. In [12], the authors showed that for smooth connectivity in heterogeneous wireless networks, the main objective of handoff is choosing the best target network from the existing network. To accomplish this goal, they designed a target network selection algorithm to improve the degree of customer satisfaction. The technique focuses on a dwell time and estimation of the received signal strength.

In [13], the authors suggested a DS-CDMA cellular network traffic model combining both soft capacity and soft handover. Network output is then measured for the blocking of calls. In [14], the authors showed that based on a pre-processed understanding of the performance profiles of the links, neural networks essentially make important handoff choices with the assessment of the quality of the links, instead of comparing the relative intensity of the signal. The results of the study and simulations showed that the suggested algorithm enhances user-perceived quality in a VoIP application communication environment by reducing both the amount of packet lost and unwanted handoffs. In [15], a handover algorithm is proposed, and its output is studied in paper. Handoff decisions are usually based on signal strength, and many artificial intelligence techniques such as Fuzzy Logic, ANN and so on are utilized lately. 


\section{INTRODUCTION TO NEURAL NETWORKS TOOLS USED FOR HANDOVER DECISION MECHANISM}

In this paper, a comparison of handoff mechanism using different tools of neural networks is presented. Here, Back propagation and Radial Basis Functions tools of neural networks are used and a comparison between the efficiency of both the tools is presented depending upon their role in taking the handover decisions with the use of matlab coding. In this section, an introduction to both the tools is given and then in the next section a comparison between them is presented.

\subsection{Back propagation neural network}

Back propagation, a compression for "in reverse proliferation of errors", is a typical strategy to train the artificial neural systems. From a perfect output, the system gains from various information sources i.e. inputs, just like the manner in which a child figures out a way to acknowledge a dog. BPNN i.e. back propagation neural network is a supervised learning strategy, and is a speculation of the delta rule. BPNN needs a dataset of the perfect output for a few inputs that makes up the training set. It is most useful for feed forward systems. Back propagation necessitates the activation operate used by artificial neurons (or "hubs") be differentiable [9]. The target of a supervised learning algorithmic program is to search a function which suits best, a collection of input to its precise output. Variety of input (i/p) and output $(\mathrm{o} / \mathrm{p})$ pattern is effortlessly well-read by single-layer neural networks additionally referred to as perceptrons. Though, these perceptrons can't be trained for many relatively simple patterns for example those who are linearly indivisible. For instance, a human being will classify an picture of an animal by recognizing many characteristics like the feel of the skin, (whether it's feathered, furry, scaled), number of limbs, the animal size and lots of alternative characteristics [16].

A single-layered neural network, however must learn a function that outputs a label exclusively using the intensity of the pixels within the image. A multi-layered network overcome this constraint because it will turn out inner representations and get trained for various characteristics in every layer [1]. The primary layer can be responsible for learning the orientations of lines victimisation the inputs from the each single picture element within the image [17]. The second layer might join the options or characteristics learned within the $1^{\text {st }}$ layer and obtain trained to acknowledge shapes like circles. With every higher layer, it learns additional characteristics. The target and inspiration for development of the rear propagation algorithm (BPNN) is to seek out a way to coach multi-layered neural networks such it will learn any random mapping of input to output Differentiable activation functions [18].

The BPNN search the least value of the error function in weight space by using the gradient descent method. The amalgamation of weights that reduces the error function is supposed to be an answer of the learning problem. As the method needs calculation of the error function gradient at every step of iteration, one should ensure the permanence and differentiability of error function. Apparently one need to utilize a type of activation function apart from the step function which is used in perceptrons, because the composite function created by consistent perceptrons is irregular. Out of all, the popular activation functions for back propagation networks is sigmoid function [19]. sc: given by the expression

$$
s_{c}(x)=\frac{1}{1+e^{-c x}}
$$

The constant $\mathrm{c}$ may be chosen indiscriminately and its reciprocal $1 / \mathrm{c}$ is known as the temperature parameter in random neural networks. The shape of the sigmoid changes in step when the value of $\mathrm{c}$ increases. The higher the values of $\mathrm{c}$, it brings the sigmoid shape like that of the step function and with the limit $\mathrm{c}=1$, the sigmoid converge to step function at origin.

Figure 3 shows architecture of back propagation. Multi-layer, Feed forward networks have the features such as: They must have in any case one hidden layer. It should have units which are non-linear. All layers must be connected between units in two sequential layers. It is not required that output units must have input units equal in number. Number of Hidden units per layer does not depend upon the number of input or output units [20].

\subsubsection{Learning in back propagation networks}

a. Learning in back propagation networks

Learning in back propagation networks: Weights initially take small and random values for each training pair (input / output). Each input unit transmits its value to every hidden unit. Every hidden unit sets its input signal and activates its output signal calculation function. 


\section{b. Back propagation stage}

Each output calculates its term of error, its own term of weight correction and its term of correction and sends it down to the layer below. Every hidden unit sums up its delta input from above and multiplies by its activation function derivative; it also calculates its own weight correcting and bias correcting term.

c. Adjusting the weights

Each output $(\mathrm{o} / \mathrm{p})$ unit updates firstly its weights and then bias and every hidden unit updates its weights and then bias term. Every training sequence is known as an epoch. The weights are reorganised in every cycle

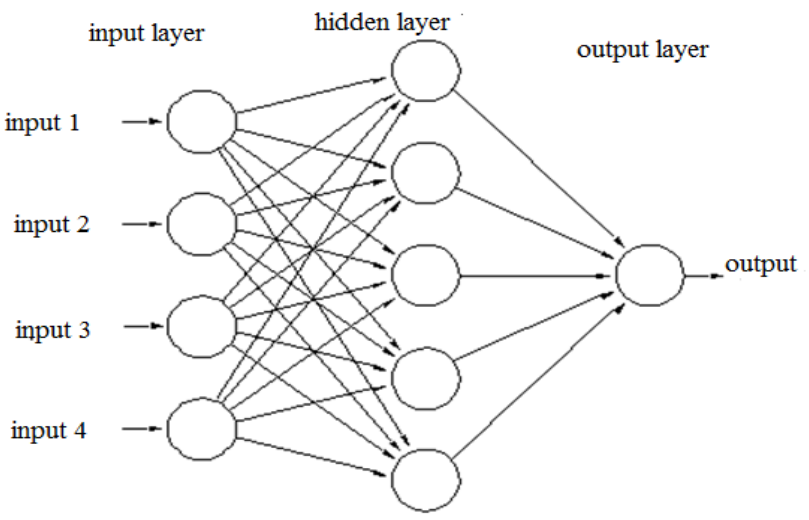

Figure 3. Architecture of back propagation

\subsection{Introduction to radial basis functions}

Moody and Darken and Broom head and Lowe, were the first persons to make use of the radial basis function $(\mathrm{RBF})$ in the system of neural networks (NN). RBF networks are the feed-forward networks practiced with the use of supervised training algorithm. These are mostly well-ordered with an individual hidden layer of units whose activation function is carefully selected among a cluster of functions known as basis function. Though they are similar to BPNN in many structures, Radial Basis Function networks has a number of advantages. They generally learn faster than BPNN. Radial basis functions are recent ways of approximating several multi variable functions. They have been recognized, examined and tested for several years now [21]. The hidden units offer functions that situate a random base for the input design [22]. There is a non-linear conversion from input space to hidden unit and linear conversion from hidden unit to the output unit. The hidden layer functions produce significant non-zero response only at times the input drops within a slightly limited region of the input [23]. Every hidden unit has its separate receptive field in input space [24]. The architecture of Radial Basis Functions is displayed in the following Figure 4.

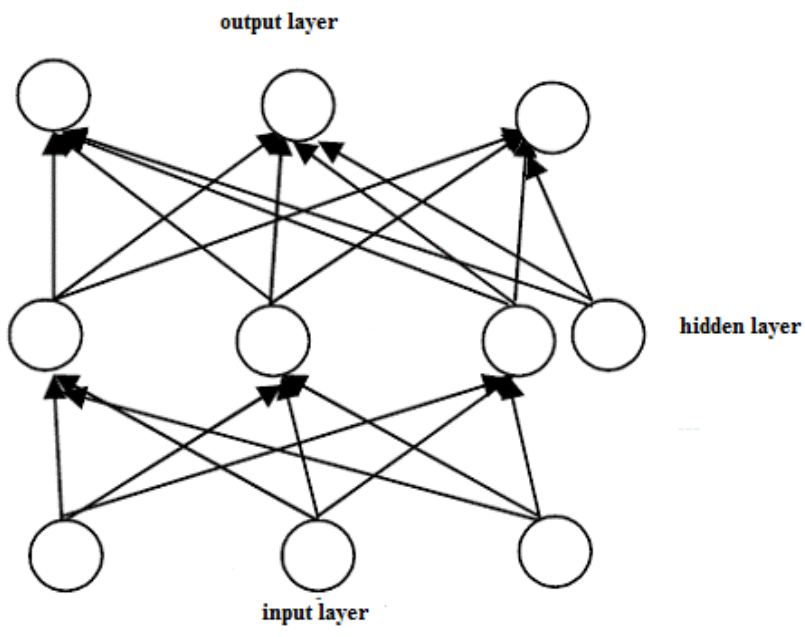

Figure 4. Architecture of radial basis function 
The main features of RBF are [25]:

- These are two layered feed-forward networks.

- The hidden nodes provide a set of Radial Basis Functions (e.g. Gaussian functions).

- The output nodes provide linear concise functions as in BPNN.

- The network training have two stages: firstly the weights from the input layers to hidden layer are specified, and then the weights from the hidden layer to output layer are specified. The training is really fast. These networks are better for doing interpolation.

\subsection{Difference between the two tools}

Comparison in the performance of BPNN and RBF networks fascinated the thoughtfulness of the many researchers. Many researchers found that the RBF defeated the BPNN in recognition of voice while some other researchers found that Multi-layer perceptron i.e. BPNN is superior to RBF in fault tolerance and allocation of resources applications [26]. Additionally approving the very fact that BPNN with sigmoid activation function is superior for classification applications [27]. BPNN with sigmoid activation didn't solely attain the simplest mean sq. error, however conjointly wished the minimum training time in on-line learning in all datasets [28]. Apart from the similarities of topologies, RBF networks and BPNN performs in a unique way. RBF networks are modest than BPNN which might have more than 3 layers, so the training method is usually quicker than BPNN. It is vital to fix accurate early states for RBF networks whereas BPNN use arbitrarily created parameters primarily [21]. The classification procedures for RBF networks and BPNN are totally different. The RBF network primarily based models are linear in parameters and thus assure convergence to their ideal values for specific network architecture. Growth of the RBF network model so needs fewer samples and fault and thus, a smaller amount of time and effort, than that desired by BP approach [29].

\section{PROPOSED APPROACH}

In this paper, handover technique based on two different tools of neural networks is shown and the best among them is selected. When the results for both the tools were drawn, it was seen that RBF is better than Back propagation of neural networks in many aspects. Let us explain this from the results and simulations of the tools. We are using two methods of neural networks i.e. Back propagation neural networks (BPNN) and radial basis function (RBF) neural network method for handover decisions in wireless communication networks.

Results of implementation have been summarized in the Table 1. From the Table 1, it is seen that classification accuracy and training time increases with increase in number of neurons in hidden layer in case of both BPNN and RBFNN. Table 1 show that classification accuracy of RBFNN is better than BPNN but the time taken by RBF is more than that of BPNN. When number of hidden layer neurons are 50 for given dataset of 2500 training input samples, corresponding classification accuracy for BPNN is $88 \%$ and training time is only 0.32 minutes and accuracy of RBFNN is $58 \%$, training time is 0.06 minutes . But when number of hidden layer neurons increases to 500, classification accuracy for BPNN is $83 \%$ and training time increases gradually to 2 minutes and 27 seconds corresponding accuracy in case of RBFNN is 90 and time taken is 11 minutes and 55 seconds. When number of hidden layer neurons increases to 500, accuracy of RBFNN increases and its corresponding value of MSE decrease (0.03). The results for the neural network tools are calculated after running the MATLAB codes assigned to back propagation and Radial Basis functions respectively. These codes are given in the appendix. Table 1 gives the performance of both the tools discussed above.

Table 1. Showing comparison between the training times calculated using RBF and back propagation

\begin{tabular}{cccccc}
\hline $\begin{array}{c}\text { Serial } \\
\text { number }\end{array}$ & $\begin{array}{c}\text { number of } \\
\text { neurons }\end{array}$ & $\begin{array}{c}\text { Accuracy } \\
\text { BPNN }(\%)\end{array}$ & $\begin{array}{c}\text { Accuracy } \\
\text { RBF }(\%)\end{array}$ & $\begin{array}{c}\text { Training time } \\
\text { BPNN in minutes }\end{array}$ & $\begin{array}{c}\text { Training Time } \\
\text { RBF in minutes }\end{array}$ \\
\hline 1 & 50 & 80 & 58 & $00: 32$ & $00: 06$ \\
2 & 100 & 79 & 64 & $00: 50$ & $00: 11$ \\
3 & 150 & 81 & 68 & $00: 56$ & $00: 19$ \\
4 & 200 & 83 & 71 & $01: 07$ & $00: 28$ \\
5 & 250 & 79 & 78 & $01: 18$ & $01: 57$ \\
6 & 300 & 87 & 81 & $01: 30$ & $03: 53$ \\
7 & 350 & 82 & 85 & $01: 42$ & $05: 43$ \\
8 & 400 & 79 & 86 & $01: 53$ & $07: 42$ \\
9 & 450 & 83 & 88 & $02: 15$ & $09: 55$ \\
10 & 500 & 83 & 90 & $02: 17$ & $11: 55$ \\
\hline
\end{tabular}


Figure 5 shows an important parameter which helps in deciding the best handover decisions. In this era where all the technologies are interworking time management in handover decisions is very important. As the user today wants to enjoy the uninterrupted services while moving from one place to another. The graph drawn above proves that the time taken by RBF in handover is practically not realisable as there is a pause of up to twelve minutes for 500 neurons. In the meantime, all the services to which the user is connected are at a halt whereas the maximum time taken by the BPNN networks for maximum number of neurons is four minutes for 500 neurons. Though the efficiency provided by RBF is much more than that of BPNN but the fact cannot be neglected that the time taken by RBF is very large.

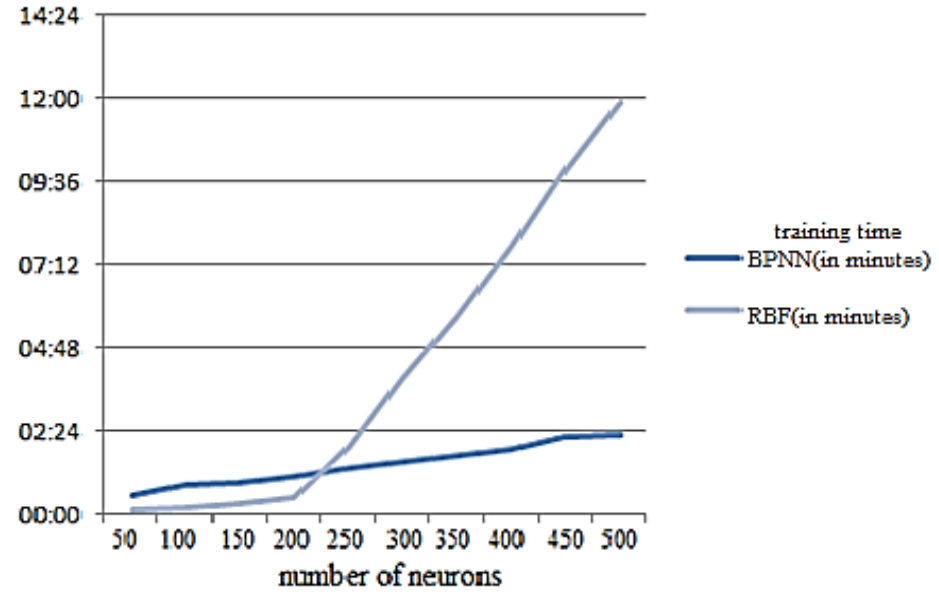

Figure 5. Training time for BPNN and RBF

The Figure 6 shows the comparison between the accuracy of RBF and BPNN and it is clearly shown that the efficiency of RBF is better than that of BPNN. For 500 neurons the accuracy for RBF reaches up to $90 \%$ whereas that of BPNN is just 83\%.In taking handover decisions, accuracy plays a vital role. Accuracy in case of handover means how accurately the handover decisions are taken when user enters the coverage area of new base station or the user intends to make use of other services rather than that service which he is currently using.

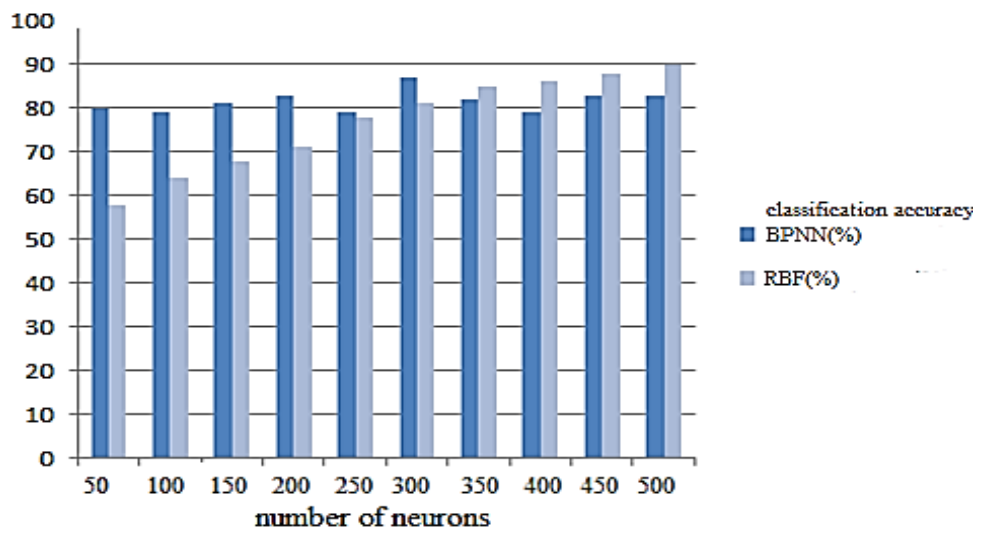

Figure 6. Graph between classification accuracy of RBF, BPNN v/s number of neurons

\section{CONCLUSION}

The handover decisions can be taken using conventional sources and soft computing techniques. In this paper, it is shown that when the neural network tools BPNN and RBF are used for taking handover decisions, RBF networks is proved to be better than BPNN in terms of accuracy but time taken by RBF is much more than that of BPNN. The performance of these classifiers is evaluated on the basis of number of 
neurons in hidden layer, training time and classification accuracy. This paper shows that RBF neural network gives better results for handover decision in wireless communication networks with classification accuracy of $90 \%$. Thus, it can be concluded that though RBF provides more accuracy but it takes a huge time for making the decisions. The future work suggested in this paper are other neural network techniques which can practically make possible the use of neural networks in handover decisions and these techniques can be C4.5 decision tree, Nave Bayes, Bayes net.

\section{REFERENCES}

[1] B. Van Quang, et al., "A survey on handoffs-lessons for $60 \mathrm{GHz}$ based wireless systems," IEEE Communications Surveys \& Tutorials, vol. 14, no. 1, pp. 64-86, 2012.

[2] X. Yan, et al., "A survey of vertical handover decision algorithms in Fourth Generation heterogeneous wireless networks," Computer Networks, vol. 54, no. 11, pp. 1848-1863, 2010.

[3] M. M. A. Khan, et al., "Interworking between WiMAX and UMTS to provide seamless services," International Journal of the Physical Sciences, vol. 5, no. 6, pp. 852-864, 2010.

[4] C. H. Lee and C. J. Yu, "An intelligent handoff algorithm for wireless communication systems using grey prediction and fuzzy decision system," 2004 IEEE International Conference on Networking, Sensing and Control, vol. 1, pp. 541-546, 2004.

[5] L. C. Paul, "Handoff/Handover Mechanism for Mobility Improvement in Wireless Communication," Global Journal of Researches in Engineering, vol. 13, no. 16, 2014.

[6] P. Mahajan, et al., "A Survey on Handover between Different Hybrid Wireless Networks," International conference of mathematical and engineering sciences, 2014.

[7] P. Mahajan, et al., "Handover based on Back propagation," International Multitrack Conference on Science, Engineering and Technical Innovations, 2014

[8] P. Mahajan, et al., "Handover Decision Mechanism in Interworking Technologies Using Radial Basis Functions," IAES International Journal of Artificial Intelligence, vol. 3, no. 2, pp. 79-83, 2014.

[9] M. T. Hagan and M. B. Menhaj, "Training feed forward networks with the Marquardt algorithm," IEEE Transactions on Neural Networks, vol. 5, no. 6, pp. 989-993, 1994.

[10] O. A. Oludolapo, et al., "Comparing performance of MLP and RBF neural network models for predicting South Africa's energy consumption," Journal of Energy in Southern Africa, vol. 23, no. 3, pp. 40-46, 2012.

[11] J. Madaan, et al., "A new method for handoff target network selection," International Journal of Robotics and Automation (IJRA), vol. 8, no. 1, pp. 36-43, 2019.

[12] J. Verma, et al., "Target Network Selection Algorithm based on Required Dwell Time Estimation," Indonesian Journal of Electrical Engineering and Informatics (IJEEI), vol. 6, no. 2, pp. 172-182, 2018.

[13] S. L. Su, et al., "Performance analysis of soft handoff in CDMA cellular networks," IEEE Journal on selected areas in communications, vol. 14, no. 9, pp. 1762-1769, 1996.

[14] T. Lin, et al., "A neural-network-based context-aware handoff algorithm for multimedia computing," $A C M$ Transactions on Multimedia Computing, Communications, and Applications (TOMM), vol. 4, no. 3, pp. 1-23, 2008.

[15] P. P. Bhattacharya, "Application of artificial neural network in cellular handoff management," in International Conference on Computational Intelligence and Multimedia Applications (ICCIMA 2007), pp. 237-241. 2007.

[16] Satishkumar, "Neural networks: a classroom approach," MC Graw Hills companies, 2004.

[17] S. Joshi and M. Borse, "Detection and Prediction of Diabetes Mellitus Using Back-Propagation Neural Network," 2016 International Conference on Micro-Electronics and Telecommunication Engineering (ICMETE), pp. 110-113, 2016.

[18] P. Wang, et al., "Applying back-propagation neural network to predict bus traffic," in 2016 12th International Conference on Natural Computation, Fuzzy Systems and Knowledge Discovery (ICNC-FSKD), pp. 752-756, 2016.

[19] P. Mahajan, et al., "Handover based on Back propagation," International Multitrack Conference on Science, Engineering and Technical Innovations, 2014.

[20] H. Leung and S. Haykin, "The complex back propagation algorithm," IEEE Transactions on Signal Processing, vol. 39, no. 9, pp. 2101-2104, 1991

[21] M. D. Buhmann, "Radial basis functions," Acta Numerica, vol. 9, pp. 1-38, 2000.

[22] L. Behera, "Modul 1 Lecture 6: Radial Basis Function Networks," Indian Institute of Technology, Kanpur, pp. $1-23$.

[23] M. Mohammadi, et al., "A hardware architecture for radial basis function neural network classifier," IEEE Transactions on Parallel and Distributed Systems, vol. 29, no. 3, pp. 481-495, 2018.

[24] Y. Shi, et al., "Action Anticipation with RBF Kernelized Feature Mapping RNN," European Conference on Computer Vision, 2018

[25] J. A. Bullinaria, "Radial Basis Function Networks: Introduction," Introduction to neural network: lecture 12, pp. 1-12, 2004

[26] R. A. Finan, et al., "Comparison of multilayer and radial basis function neural networks for text-dependent speaker recognition," Proccedings of International Conference on Neural Networks, vol. 4, pp. 1992-1997, 1996.

[27] T. Xie, et al., "Comparison between Traditional Neural Networks and Radial Basis Function Networks," IEEE International Symposium on Industrial Electronics, pp. 1194-1199, 2011. 
[28] E. A. M. A. Shenouda, "A quantitative comparison of different MLP activation functions in classification," Advances in Neural Networks, pp. 849-857, 2006.

[29] R. B. Santos, et al., "Comparison Between Multilayer Feed forward Neural Networks and a Radial Basis Function Network to Detect and Locate Leaks in Pipelines Transporting Gas," Chemical Engineering Transactions, vol. 32, pp. 1375-1380, 2013

\section{BIOGRAPHIES OF AUTHORS}

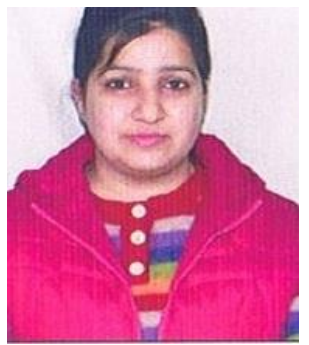

Payal Mahajan, Research Scholar in Department of Electrical Engineering, Jamia Millia Islamia University. Research interests: fuzzy logic, wireless communication, mobile networks, neural networks, optimization techniques. Email: payal27mahajan@gmail.com

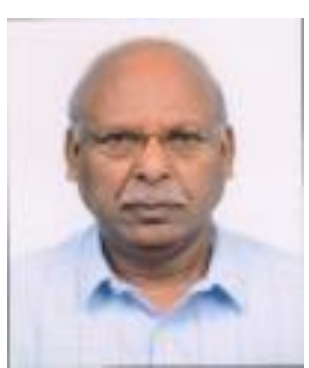

Prof. Zaheeruddin, Senior professor in Department of Electrical Engineering, Jamia Millia Islamia University. Research interests: fuzzy logic, soft computin, mobile networks, artificial Intelligence neural networks, optimization techniques. Email: zaheeruddin@jmi.ac.in 